\title{
sciendo
}

\author{
BULGARIAN ACADEMY OF SCIENCES
}

CYBERNETICS AND INFORMATION TECHNOLOGIES • Volume 19, No 2

Sofia $2019 \quad$ Print ISSN: 1311-9702; Online ISSN: 1314-4081

DOI: $10.2478 /$ cait-2019-0018

\section{Genetic Algorithms vs. Knowledge-Based Control of PHB Production}

\author{
Marius Olteanu ${ }^{1}$, Nicolae Paraschiv ${ }^{1}$, Petia Koprinkova-Hristova ${ }^{2}$ \\ ${ }^{1}$ Petroleum-Gas University of Ploiesti, Ploiesti, Romania \\ ${ }^{2}$ Institute of Information and Communication Technologies, Bulgarian Academy of Sciences, 1113 Sofia, \\ Bulgaria \\ E-mails:molteanu@upg-ploiesti.ronnparaschiv@upg-ploiesti.ro pkoprinkova@bas.bg
}

Abstract: The paper proposes an approach using Genetic Algorithm (GA) for development of optimal time profiles of key control variable of Poly-HydroxyButyrate $(P H B)$ production process. Previous work on modeling and simulation of PHB process showed that it is a highly nonlinear process that needs special controllers based on human experience, as such fuzzy logic controller proved to be a good choice. Fuzzy controllers are not totally replaced, due to the specific process knowledge that they contain. The achieved results are compared with previously proposed knowledge-based approach to the same optimal control task.

Keywords: Genetic algorithm, fuzzy logic, PHB production, optimization.

\section{Introduction}

Genetic Algorithms (GA) are a technique of Artificial Intelligence that solve optimization problems by exploring and searching the space of possible solutions (encoded as chromosomes) by genetic operators like selection, crossover and mutation $[15,25]$.

Being a heuristic optimization method, its theoretic foundation explains only partially (schemata representation in building block hypothesis) this results that in many situations overcomes other methods [12]. An important aspect of practical implementations of GA is in the choices that the designer has to make with respect to the codification of chromosomes and the genetic operators tailored to the specific problem. By far GAs have numerous applications in process control [38], non-linear models identification [31,35] as well as data clustering [24].

Fuzzy logic is another intelligent technique converting qualitative knowledge presented as a set of linguistic rules into quantitative input-output relations [39]. It allows numerical formalization of accumulated human experience and experts' optimal solutions that can be hardly obtained analytically. Since its invention by Prof. Zadeh in 1965 fuzzy logic found numerous application in intelligent systems [33, 37] and particular in intelligent control [2, 34]. 
Nowadays variety of hybrid optimization approaches combining GAs as well as other heuristic optimization techniques and fuzzy logic and neural networks were developed, among which we mention only some representative examples from last few years $[1,4-6,10,11,14,22,23,27,28,32]$.

The aim of the present paper is to compare both intelligent techniques on an example of optimal control of a highly non-linear microbiological process for production of a biodegradable polymer poly- $\beta$-hydroxybutyrate (PHB).

The paper is organized as follows: next section gives brief description of the PHB production process peculiarities and the optimal control task that has to be solved; section three presents accumulated expertise in PHB optimization and control and proposed previously knowledge-based optimal solution; next GA are introduced and their application to the same optimization task is presented; next obtained by both intelligent techniques optimal time profiles of the key control variables were presented and compared; the paper finishes with conclusions and directions for future work.

\section{PHB production process}

\subsection{Process model}

PHB production belongs to the class of mixed-culture microbiological processes in which several microorganisms shearing common living resources (food and environmental conditions) cooperate and produce some target product as a result of conversion of feeding substrates and intermediate metabolites. In particular, in PHB production sugars (glucose) and nitrogen source foods were converted to the intermediate product lactate by the microorganism L. delbrueckii - Lactobacillus delbrueckii, until 2014 known as Lactobacillus bulgaricus, first identified in 1905 by the Bulgarian doctor Stamen Grigorov; next lactate was converted further to target product PHB (biodegradable polymer used as thermoplastic in food and drug industry) by the microorganism $R$. eutropha. The two strains have different demands on concentration of Dissolved in cultural medium Oxygen (DO). Besides, the first one produces additional food source (lactate) for the second one.

The process was modelled by [36] the following nonlinear ordinary differential equations system:

$$
\begin{gathered}
\frac{d X_{1}}{d t}=\mu_{1}(S, P, \mathrm{DO}) X_{1}-\frac{F_{S}}{V} X_{1}-\frac{F_{n}}{V} X_{1}, \\
\frac{d S}{d t}=-v_{1}(S, P, \mathrm{DO}) X_{1}+\frac{F_{S}\left(S_{F}-S\right)}{V}-\frac{F_{n}}{V} S, \\
\frac{d P}{d t}=\sigma_{1}(S, P, \mathrm{DO}) X_{1}-v_{2}(P, \mathrm{DO}, N) X_{2}-\frac{F_{S}}{V} P-\frac{F_{n}}{V} P, \\
\frac{d X_{2}}{d t}=\mu_{2}(P, \mathrm{DO}, N) X_{2}-\frac{F_{S}}{V} X_{2}-\frac{F_{n}}{V} X_{2},
\end{gathered}
$$




$$
\begin{gathered}
\frac{d N}{d t}=-v_{3}(P, \mathrm{DO}, N) X_{2}+\frac{F_{n}\left(N-N_{F}\right)}{V}-\frac{F_{S}}{V} N, \\
\frac{d Q}{d t}=\sigma_{2}(N) X_{2}-\frac{F_{S}}{V} Q-\frac{F_{n}}{V} Q, \\
\frac{d V}{d t}=F_{S}+F_{n} .
\end{gathered}
$$

They represent the dynamics of the main process state variables: $X_{1}$ - concentration of $L$. delbrueckii cells; $X_{2}$ - concentration of $R$. eutropha cells; $S$ - glucose concentration; $P$ - lactate concentration; $N$ - nitrogen source concentration; and $Q$ - product, i.e., PHB concentration. The specific growth, production and consumption rates $\mu_{1}, \mu_{2}, v_{1}, v_{2}, v_{3}, \sigma_{1}$, and $\sigma_{2}$ depend on the process state variables as well as on the DO in the cultural medium.

The dissolved oxygen concentration was maintained at its set point by changing the agitation speed and/or the air flow rate into reactor. Both food sources (glucose and nitrogen source) concentrations were controlled independently from each other by their feeding rates $F_{S}$ and $F_{n}$, respectively.

\subsection{Control problems and expert knowledge}

The main purpose of process control strategy is to maximize the target product outcome accounting for the contradicting needs and mutual relations of both microorganisms in the culture. By now there are known several approaches to this problem. In [36] different control strategies were exploited separately or in combination: to maintain the lactate concentration at a given optimal level using dissolved oxygen concentration as control variable, to maintain the glucose concentration at a given optimal level by its feeding rate, to change the set point of the glucose concentration according to the lactate concentration deviation from its set point. In [16] it was proposed to monitor the lactate production and consumption rates in order to determine the needs of the two microorganisms and depending on them to feed glucose or to change dissolved oxygen concentration. Another approach is adaptive control strategy proposed in [29] that determines the optimal glucose feeding rate based on the known from [36] optimal level of the lactate concentration or glucose concentration and monitoring of the second microorganism's concentration and lactate [30]. In [9] it was proposed to maximize the process productivity by controlling the mixing intensity. In [26] an intelligent approach to optimization of the glucose and ammonium time profiles is proposed. It uses neural networks for process model and feed-back controller. In [17] fuzzy control approach is proposed that combines the expert knowledge about the lactate concentration dependence on the set points of dissolved oxygen and glucose concentrations. Further in [19] the application of experts' knowledge about the process control is summarized and extended. A combined fuzzy rule based control system is applied and compared with previously used control approaches. In [18] the neural network approach called Adaptive Critic Design (ACD) was applied to synthesis of sugar's concentration optimal time profile for the process. In [20] the same approach was extended to 
synthesis of optimal time profiles of all three main substrates (sugar, nitrogen source and dissolved oxygen). In [21] that approach is investigated further.

The main expert knowledge about proper control of PHB production claims [36] that the most important thing is to maintain the key process variables - intermediate metabolite (lactate) and second food source (nitrogen) as well as dissolved oxygen concentration - at optimal levels can be summarized as follows:

Although lactate is needed for target product synthesis, at high concentrations it suppresses second strain growth. It was experimentally discovered [36] that there is an optimal steady state value of lactate concentration that should be maintained during the process once it is reached.

High nitrogen source concentrations inhibit growth of both strains so it should not be increased over some limit. However, nitrogen is needed for target product synthesis.

Lower dissolved oxygen concentrations enhance growth of the first strain and thus the lactate production rate while higher dissolved oxygen concentrations enhance the cell growth of the second strain and hence the lactate consumption rate.

Thus the process has two stages: from the beginning until increase of lactate up to its optimal level and steady state with almost constant concentration of lactate. Both stages have also different demands on nitrogen source: during the first stage both strains growth must be enhanced by relatively higher nitrogen source concentration while during the second stage the needs of nitrogen source are limited and it must be kept at minimal level so as to maintain second strain growth and thus to support target product synthesis.

Hence the proper balance can be achieved by accounting for the process stage as well as for deviations of lactate concentration from its optimal set point during the second stage. Since the lactate concentration depends both on its production rate by L. delbrueckii and its consumption rate by R. eutropha, each of those specific kinetic rates have to be controlled by changing the set points of the controllers for the substrate and dissolved oxygen concentrations for the lactate production rate and the nitrogen and dissolved oxygen concentrations for the lactate consumption rate.

Following available expert knowledge, in previous investigations [19] three knowledge-based control systems were developed: for dissolved oxygen concentration set point DO*, for substrate (sugars) concentration set point $S^{*}$ and for nitrogen source concentration set point $N^{*}$. The overall process control scheme is shown on Fig. 1.

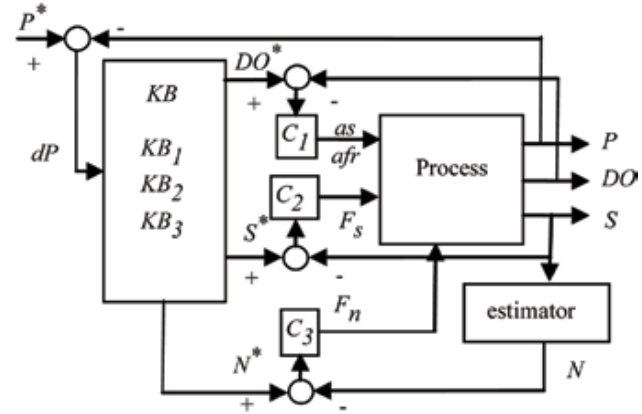

Fig. 1. Knowledge-based control scheme of PHB production process 
Here $C_{1}, C_{2}$ and $C_{3}$ denote the controllers for the dissolved oxygen concentration (as - agitation speed; afr - air flow rate), glucose and nitrogen concentration respectively. It is supposed that the nitrogen concentration was measured by software sensor using available on-line measurements [29].

In all three fuzzy rule bases are of Mamdani type and the fuzzy values of linguistic variables defined by bell-shaped or triangular membership functions depending on the given rule base [19]. The crisp output of was obtained by centroid defuzzyfication method [2]. The membership functions parameters and shapes are determined intuitively and by simulation investigations [19]. The main "art" in determining membership functions parameters was based on the experts' knowledge about the process and understanding of membership functions' parameters influence on the final inference result from the given fuzzy rule base [19], e.g., the shape of the knowledge base output surface and hence the overall system's dynamics.

\section{Genetic algorithm for optimization}

In present work GA was applied to determine the optimal time profile of only one key variable - set point of Dissolved Oxygen (DO*). It replaced first fuzzy rule base while the other two fuzzy rule bases intended to control the Sugar $\left(S^{*}\right)$ and Nitrogen source $\left(N^{*}\right)$ set points remained the same as in [19].

The solutions (chromosomes) contain the time profile of dissolved oxygen set point $\mathrm{DO}^{*}(\mathrm{t}), t=4, \ldots, T$. They consisted of real values restricted in the interval $\mathrm{DO}^{*}{ }_{\min }=0.5, \mathrm{DO}^{*}{ }_{\max }=3.0$. The length of the vector is determined by the simulation time starting after inoculation of the second strain into reactor (4th hour from the beginning of first strain cultivation) until the end of process $(T=30 \mathrm{~h})$, i.e., the chromosomes were vectors of 26 real numbers.

At the beginning of optimization, chromosomes' elements were generated randomly within allowed interval. The population size (popsize $=50$ ) was determined experimentally in order to achieve affordable computing efforts and running time of the algorithm.

The fitness function

aim was to favour solutions achieving higher product concentrations as well as to penalize the solutions that achieve lactate concentrations far from the optimal set point $\left(P^{*}=3.87 \mathrm{~g} / \mathrm{l}\right)$. The moment when penalty starts was experimentally determined to $t_{P}=20 \mathrm{~h}$. The penalty weight $k=0.1$ was used to increase importance of the first term in fitness function.

Selecting the best members of the current population of solutions is a first important step of the GA that influences all the subsequent steps and the possible outcome. Two selection approaches were compared:

Rank selection is based on sorting the population members according to their fitness value and choosing a specified number of first best members (usually $40-50 \%$ of entire population).

In tournament selection a number of random members (specified in the operator) are chosen to compete in the tournament and the best one is selected. The operator is 
implemented such that once a member is selected, it will not be selected for further tournaments [13].

Between the two selection methods, results given by the rank selection methods were much more suitable due to its reduced utilization of random numbers.

The new generation of solutions was produced mainly by the crossover operator. Two methods were implemented by the present algorithm: fixed single point crossover and arithmetic mean. In either case, the crossover operator is applied with a variable probability, usually $p_{\text {cross }} * 0.6-0.8$, that is, the operator is applied to a number of $p_{\text {cross }}{ }^{*}$ popsize members.

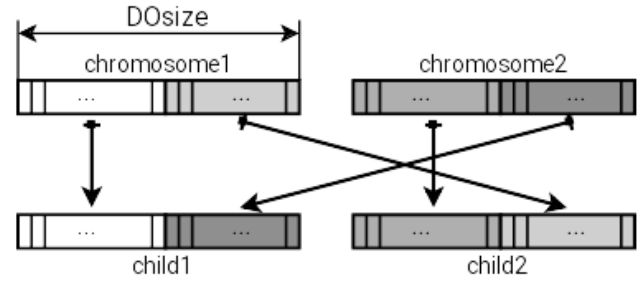

Fig. 2. Single point crossover operator

Fixed single point crossover chooses randomly two members of the population and switches the two halves of both parents (the crossover point is actually located in the middle) in order to generate two new offspring that will compose the new population.

Arithmetic mean crossover operator is usually applied in the case of real coding of the solution, having very good results. Two members of the population are randomly chosen and their values are mediated resulting in a single offspring that will be part of the new population [3]

After a sufficient number of tests, it resulted that a better exploration of the solution space is possible with the aid of the single point crossover operator despite the fact that real encoding is usually associated with the arithmetic mean operator.

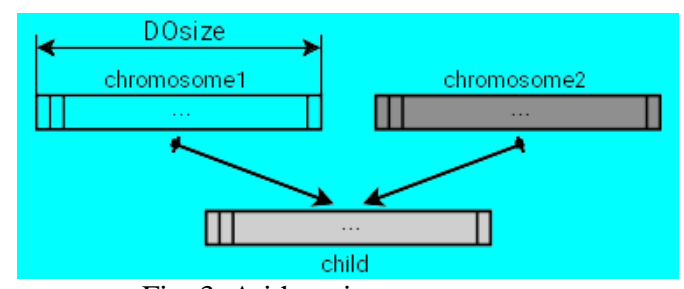

Fig. 3. Arithmetic mean operator

In many situations the only possibility of introducing variability (and also to avoid premature convergence of the population) in the population of solutions is to mutate some of its members.

The mutation operator randomly choose a specified number of members (specified by the mutation probability $p_{\text {mut }}$ ) for which a number of values of the DO* vector is randomly changed to a value in the range $\left[\mathrm{DO}^{*}{ }_{\min }, \mathrm{DO}^{*}\right.$ max $]$. The number of values is expressed as a fraction of the vector, or strength (for example strength $=0.4)[3]$. 


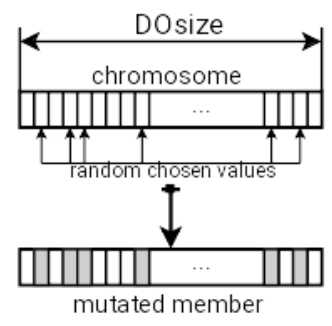

Fig. 4. Mutation operator

The GA ran many times in order to establish the best stopping criteria and also the dispersion of the results. A reasonable number of generations $n_{\text {gen }}=50$ proved to be a good compromise between the running time of the algorithm and convergence.

Table 1. Genetic algorithm multiple runs

\begin{tabular}{|c|c|c|c|c|c|c|c|}
\hline No & $\begin{array}{c}\text { Number of } \\
\text { generations }\end{array}$ & $\begin{array}{c}\text { Members of } \\
\text { population }\end{array}$ & $\begin{array}{c}\text { Selection } \\
p_{\text {sel }}\end{array}$ & $\begin{array}{c}\text { Crossover } \\
p_{\text {cross }}\end{array}$ & $\begin{array}{c}\text { Mutation } \\
p_{\text {mut, }} \\
\text { strength }\end{array}$ & Time & Fitness \\
\hline 1 & 30 & 30 & 0.4 & 0.8 & $0.25,0.4$ & $1^{\prime} 56.6^{\prime \prime}$ & 1202.72 \\
\hline 2 & 30 & 30 & 0.4 & 0.8 & $0.25,0.4$ & $2^{\prime} 25.4^{\prime \prime}$ & 1194.08 \\
\hline 3 & 30 & 12 & 0.4 & 0.8 & $0.25,0.4$ & $35.88^{\prime \prime}$ & 1175.39 \\
\hline 4 & 50 & 50 & 0.5 & 0.8 & $0.30,0.4$ & $4^{\prime} 33.8^{\prime \prime}$ & $\mathbf{1 5 2 4 . 8 0}$ \\
\hline 5 & 100 & 100 & 0.5 & 0.8 & $0.3,0.4$ & $18^{\prime} 5.58^{\prime \prime}$ & 1439.70 \\
\hline 6 & 50 & 50 & 0.5 & 0.8 & $0.3,0.4$ & $4^{\prime} 26.2^{\prime \prime}$ & 1515.50 \\
\hline 7 & 50 & 50 & 0.5 & 0.8 & $0.1,0.2$ & $4^{\prime} 25.7^{\prime \prime}$ & 1260.27 \\
\hline
\end{tabular}

It is obvious from data presented in Table 1 that increasing the number of population members or the number of generations did not imply an increase in the final product (maximum fitness function value). Also, increasing the number of members in the population slows down significantly the algorithm. The highest fitness value was achieved using GA with parameters shown on Table 1, Case 4 .

For a future possible improvement of the PHB process control optimization by Genetic Algorithms, a much bigger set of experiments should be done, taking into account all the tuning parameters of the algorithm and even increasing them. Having a parallel implementation on more powerful computers, it would be possible to replace the fuzzy controllers also. In this sense, a comparison has been done between implementations of the simulation process on different software packages of the same process and fuzzy algorithms, the results being summarized in Table 2. In order to have as short as possible running time, it is imperative that the fitness function be evaluated as fast as possible which results in a necessity of optimized code for the process model and fuzzy controllers.

Table 2. The identification results

\begin{tabular}{|c|c|c|c|}
\hline No & Software package & Time & PC hardware \\
\hline 1 & GNU Octave v.4.0.3 & $105.08^{\prime \prime}$ & $\begin{array}{c}\text { Linux openSuSe 42.3 Leap } \\
\text { i7-7500U@3.5GHz }\end{array}$ \\
\hline 2 & Matlab R2009b 7.9.0 & $6.07^{\prime \prime}$ & $\begin{array}{c}\text { Windows 8.1 } \\
\text { i3-4130@ 3.4GHz }\end{array}$ \\
\hline 3 & $\begin{array}{c}\text { Intel XE Composer Studio 2018 } \\
\text { Intel C++ compiler }\end{array}$ & $0.1^{\prime \prime}$ & $\begin{array}{c}\text { Linux openSuSe 42.3 Leap } \\
\text { i7-7500U@3.5GHz }\end{array}$ \\
\hline
\end{tabular}


The simulation programs were coded in the $\mathrm{C}$ language under Linux operating system (openSuSE 42.3, x86_64) on Intel i7 7500U@3.5GHz microprocessor. Two different $\mathrm{C}$ compilers were used with slightly different performance time: Oracle Sun "sunce" (Sun C 5.13 Linux_i386 2014/10/20) and Intel C compiler "icc" (icc version 18.0.0 - Intel XE Composer Studio 2018). The Intel compiler proved to be the better one, with a speedup of about $20 \%$. The GNU GSL library version 2.4 mathematical routines for numerical integration of the system of differential equations were used.

\section{Comparison with knowledge-based control strategy}

The obtained by GA optimal time profile of the dissolved oxygen concentration set point was compared with that generated by extracted from expert knowledge fuzzy rule base [19]. Fig. 4 presents both time profiles of DO* while Fig. 5 represents the corresponding process outcome (target product concentration time trend). The next Figs 6-10 compare time trends of the rest of the process state variables obtained by both control strategies (fuzzy and GA).

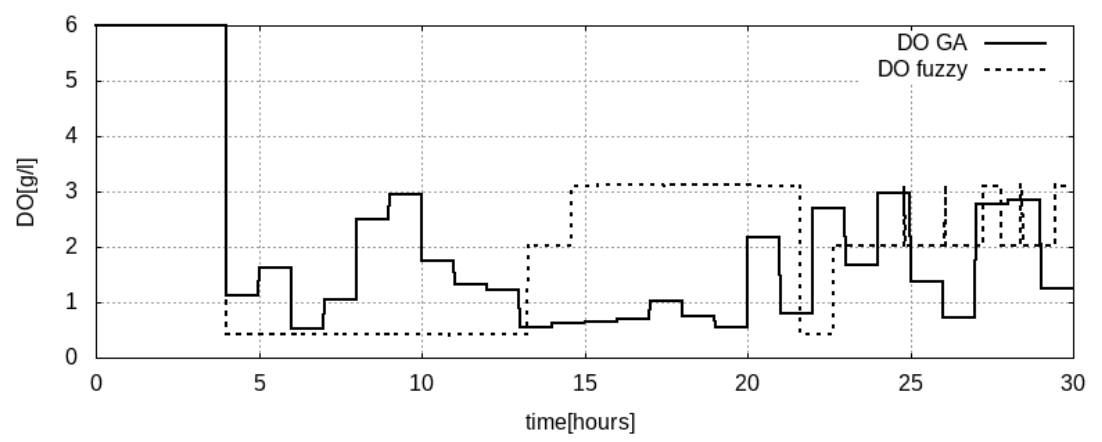

Fig. 5. Manipulated variable DO for a running time of 30 hours

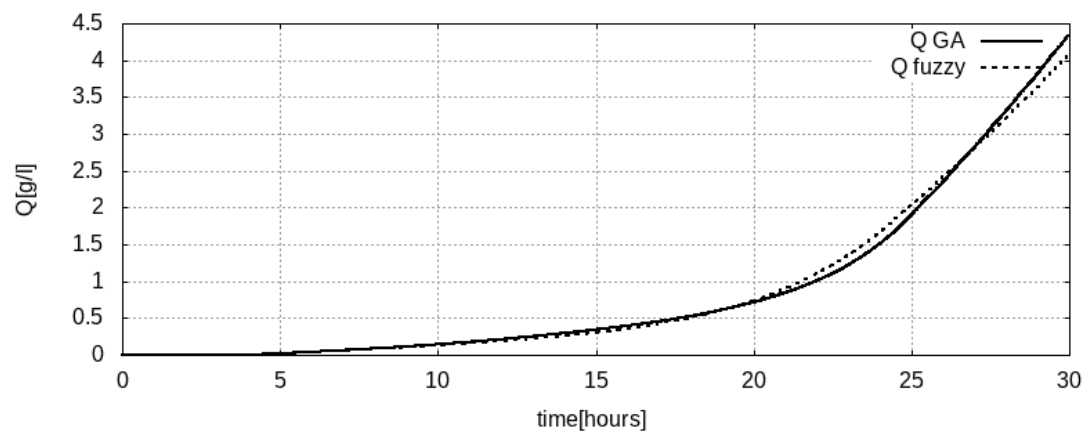

Fig. 6. Product of PHB for a running time of 30 hours

The GA achieved $6.13 \%$ higher PHB concentration at the end of simulation in comparison with knowledge-driven control strategy. The DO* profiles however differ significantly.

While fuzzy logic follows expert prescription to keep dissolved concentration as low as possible at the first stage of the process (after inoculation of second strain 
at $4 \mathrm{~h}$ until $15 \mathrm{~h}$ of simulation) in order to foster growth of the first strain until the lactate concentration reaches its optimal level, the GA tries to foster growth of the second strain from the beginning in attempt to increase product outcome. From Figs 7 and 10 we observe that while GA control strategy achieved higher concentration of the second strain, the fuzzy strategy yielded higher concentration of the first strain cells. This led (Fig. 9) to higher lactate concentrations in the case of knowledge-based control so that it's optimal set point $P^{*}$ was approached earlier than in the case of GA synthesized control. This explains the significant difference of set point time profiles of sugar (Fig. 8) and nitrogen source (Fig. 11) concentrations generated by other two knowledge-based controllers whose aim is to decrease both of $S^{*}$ and $N^{*}$ after approaching of the optimal steady state $P^{*}$.

During the second stage of the process (after $15 \mathrm{~h}$ until $20 \mathrm{~h}$ ) the GA strategy obviously "discovers" that without lactate the second strain could not grow anymore and starts to promote first strain by decreasing DO*. On the opposite, the knowledgebased approach favours the second strain since now it has enough food (lactate) to produce target product. Due to that the concentrations of both strains begin to grow faster in the case of GA control strategy (Figs 7 and 10). Since now lactate is closer to its optimal value (Fig. 9), fuzzy logic controllers decrease $S^{*}$ and $N^{*}$ (Figs 8 and 11) accordingly.

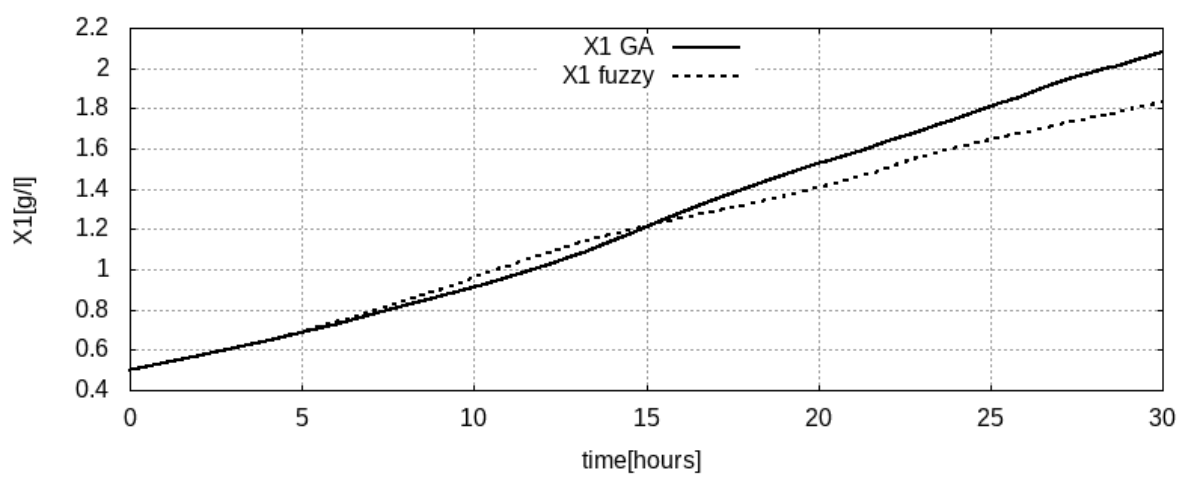

Fig. 7. Concentration of L.delbrueckii for a running time of 30 hours

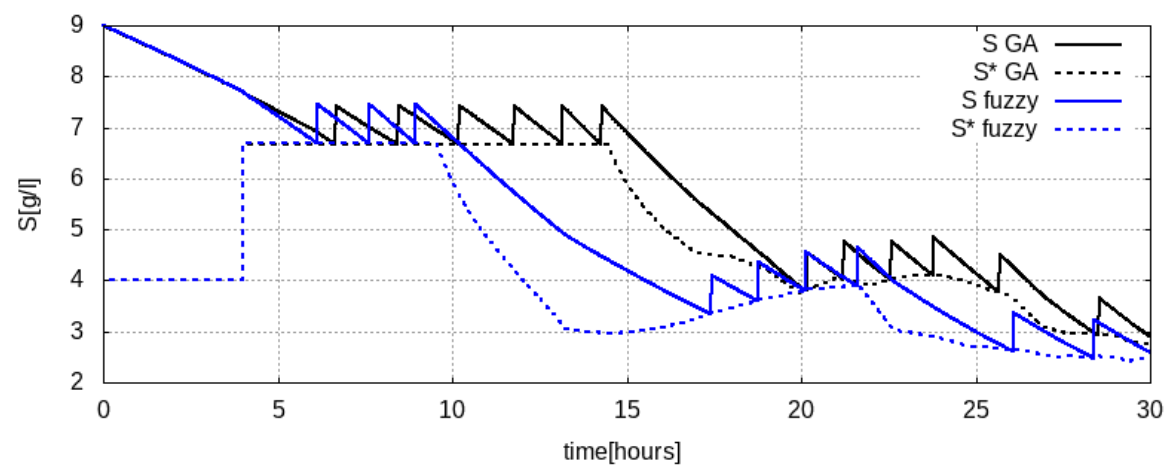

Fig. 8. Concentration of glucose for a running time of 30 hours 


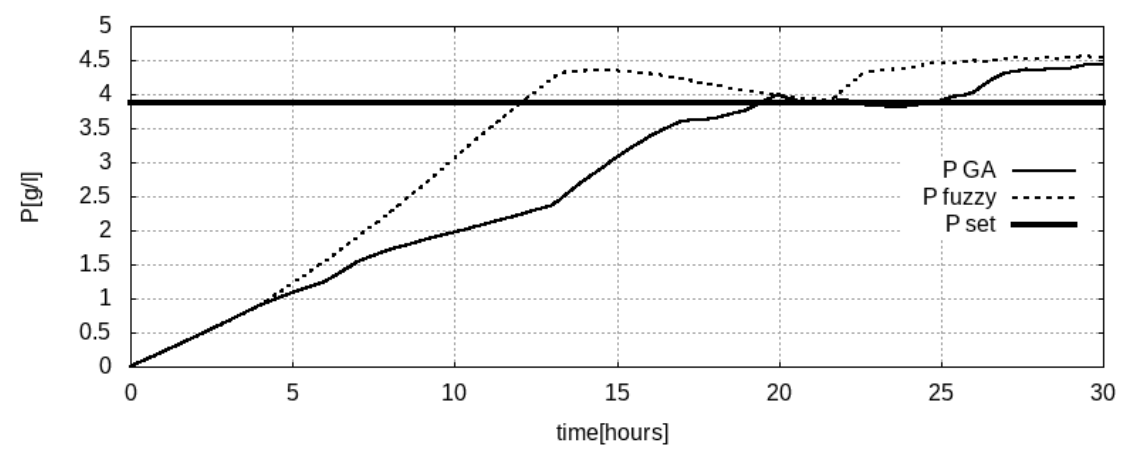

Fig. 9. Concentration of lactate for a running time of 30 hours

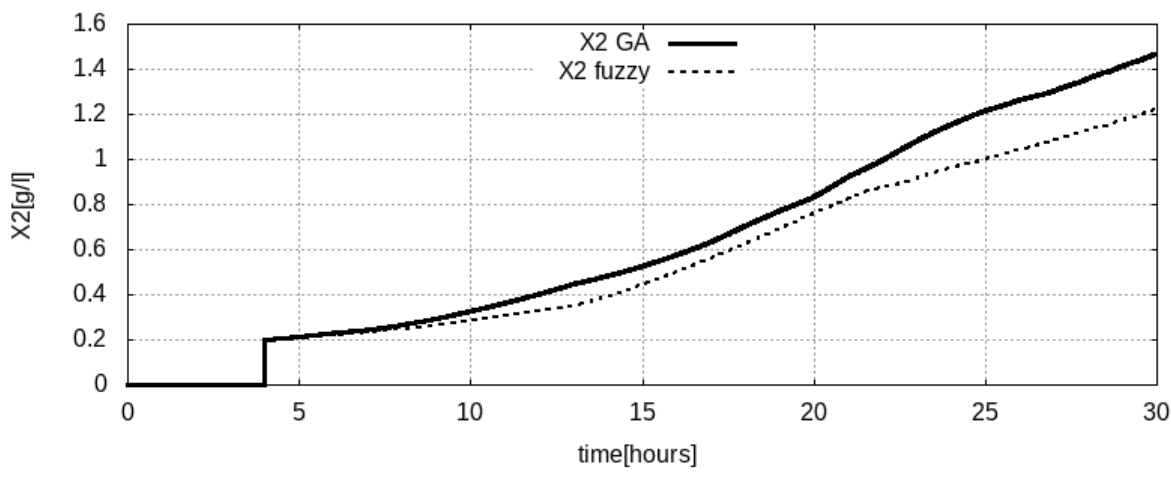

Fig. 10. Concentration of R.eutropha for a running time of 30 hours

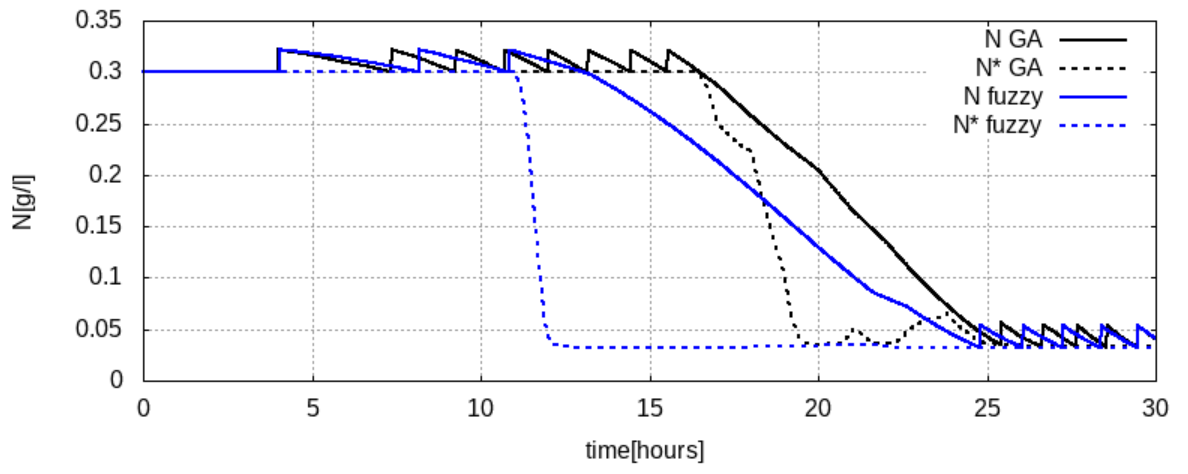

Fig. 11. Concentration of $\mathrm{NH}_{3}$ for a running time of 30 hours

At the final stage of the process (after $20 \mathrm{~h}$ ) both solutions try to keep the balance between oxygen demands of both strains switching between high and low oxygen concentrations and here GA outperforms the knowledge-based strategy achieving higher final outcome. Since the penalty term of fitness function is now acting, the GA derived DO* profile is able to maintain lactate at its optimal level longer than fuzzy control strategy (Fig. 9). However, by the end of the process both strategies fail to keep lactate close to its optimal value. During that final stage both fuzzy logic controllers of $S^{*}$ and $N^{*}$ have similar behaviour. 
In conclusion, we demonstrated the ability of GA to solve heuristically a complex control problem of keeping the balance between oxygen demands of both strains in the culture. It also "discovered" a control strategy during the first stage of the process different from experts' opinion that however appeared better with respect to final process outcome. Another reason for this result is late switching on of the penalty term of the fitness function that led to slower reaching of the process steady state but surprisingly to increased productivity.

\section{Conclusion}

The comparison of both intelligent approaches to nonlinear process control revealed their advantages and shortcomings as follows:

The knowledge-based approach allows extracting and formalizing accumulated expert knowledge about proper control of nonlinear processes and it outperformed the GA optimization strategy during the process's first stage.

Genetic algorithm however demonstrated ability to "extract" process demands during second stage thus achieving better final outcome of the process.

Combination between both approaches in future would allow to combine available knowledge with ability of GA to refine and advance it heuristically.

\section{References}

1. B a n s a 1, N., P. K a u r. A Novel Approach to Fuzzy Model Identification Based on Bat Algorithm. - International Journal of Applied Metaheuristic Computing, Vol. 10, 2019, No 2, pp. 93-108.

2. Driankov, D., H. Helle nd o orn, M. Re in frank. An Introduction to Fuzzy Control. Berlin, Heidelberg, Springer-Verlag, 1993.

3. D u mitre s c u, D. Algoritmi Genetici si Strategii Evolutive - Aplicatii in Inteligenta Artificiala Si in Domenii Conexe. Cluj-Napoca, Editura Albastra, 2006.

4. Elbaz, K., S.-L. She n, A. Zhou, D.-J. Yu a n, Y.-S. Xu. Optimization of EPB Shield Performance with Adaptive Neuro-Fuzzy Inference System and Genetic Algorithm. - Applied Sciences, Switzerland, Vol. 9, 2019, No 4, Art. No 780.

5. Es pa nol a, J. L., A. A. B a nd a la, R. R. P. Vi c e rra, E. P. D a di o s. Design of a FuzzyGenetic Controller for an Articulated Robot Gripper. - In: IEEE Region 10 Annual International Conference, Proceedings/TENCON, October 2018, Art. No 8650431, 2019, pp. 1701-1706.

6. Fidanova S., M. Paprzycki, O. Roeva. Hybrid GA-ACO Algorithm for a Model Parameters Identification Problem. - In: Proc. of 2014 Federated Conference on Computer Science and Information Systems, Vol. 2, 2014, pp. 413-420.

7. Galaviz-Aguilar, J. A., P. Roblin, J. R. Cárden a s-Valdez, E. Z-Flores, L. Tr u j i 11 o, J. C. N u ñ e z-Pér e z, O. S c h ü t z e. Comparison of a Genetic Programming Approach with ANFIS for Power Amplifier Behavioral Modeling and FPGA Implementation. - Soft Computing, Vol. 23, 2019, No 7, pp. 2463-2481.

8. Gá m e z, J. C., D. G a r c í a, A. G on zále z, R. Pér e z. An Approximation to Solve Regression Problems with a Genetic Fuzzy Rule Ordinal Algorithm. - Applied Soft Computing Journal, Vol. 78, 2019, pp. 13-28.

9. G a n d u ri, V.S. R. K., S. G h o s h, P. R. P a t n a i k. Mixing Control as a Device to Increase PHB Production in Batch Fermentations with Co-cultures of Lactobacillus Delbrueckii and Ralstoniaeutropha. - Process Biochemistry, Vol. 40, 2005, pp. 257-264. 
10. G e o r g i e v a, P. Genetic Fuzzy System for Financial Management. - Cybernetics and Information Technologies, Vol. 18, 2018, No 2, pp. 20-35.

11. Go 1 a, A., G. Kło s o w s ki. Development of Computer-Controlled Material Handling Model by Means of Fuzzy Logic and Genetic Algorithms. - Neurocomputing, Vol. 338, 2019, pp. 381-392.

12. Gold b e r g, D. Genetic Algorithms in Search, Optimization and Machine Learning. - Reading MA, Addison-Wesley Professional, 1989.

13. Goya 1, A., P. A. Sourav, P. Ka ly an araman. Application of Genetic Algorithm Based Intuitionistic Fuzzy k-Mode for Clustering Categorical Data. - Cybernetics and Information Technologies, Vol. 17, 2017, No 4, pp. 99-113.

14. H a u p t, R. L., S. E. H a u p t. Practical Genetic Algorithms. 2nd Edition. Wiley Interscience, 2004.

15. Holla nd, J. H. Adaptation in Natural and Artificial Systems: An Introductory Analysis with Applications to Biology, Control and Artificial Intelligence. Cambridge, MA, USA, MIT Press, 1992.

16. Ignatova, M., V. Lyubenova. Adaptive Control of Fed-Batch Processes for Poly- $\beta$-Hydroxybutyrate Production by Mixed Culture, Compt. Rend. Acad. bulg. Sci., Vol. 60, 2007, No 5, pp. 517-524.

17. Ko prink ova-H rist ov a, P. Fuzzy Control Approach to Mixed Culture Cultivation for PHB Production Process. - In: Proc. of International Conference Automatics and Informatics'07, 3-6 October 2007, Sofia, Bulgaria, pp. III-65-III-68.

18. K o p r i n k o v a-H r i s t o v a, P. ACD Approach to Optimal Control of Mixed Culture Cultivation for PHB Production Process - Sugar's Time Profile Synthesis. - In: Proc. of IEEE Intelligent Systems IS'08, Methodology, Models, Applications and Emerging Technologies, 6-8 September 2008, Varna, Bulgaria, Vol. II, pp. 12-29-12-32.

19. Koprinkova-Hristova, P. Knowledge-Based Approach to Control of Mixed Culture Cultivation for PHB Production Process. - Biotechnology and Biotechnological Equipment, Vol. 22, 2008, No 4, pp. 964-967.

20. Koprinkova-Hristova, P., G. Palm. Adaptive Critic Design with ESN Critic for Bioprocess Optimization. - Lecture Notes in Computer Science, Vol. 6353, 2010, pp. 438-447.

21. Ko p ri n k o va-H r i s t o v a, P., G. Ko s t o v, S. P o p o va. Intelligent Optimization of a Mixed Culture Cultivation Process. - Int. J. Bioautomation, Vol. 19, 2015, No 1, pp. S113-S124.

22. Ko sh i y a m a, A. S., R. T a n s che it, M. M. B. R. V e 11 a s c o. Automatic Synthesis of Fuzzy Systems: An Evolutionary Overview with a Genetic Programming Perspective. - Wiley Interdisciplinary Reviews: Data Mining and Knowledge Discovery, Vol. 9, 2019, No 2, Art. No e1251.

23. K u o, R. J., T. P. Q. N g u y e n. Genetic Intuitionistic Weighted Fuzzy k-Modes Algorithm for Categorical Data. - Neurocomputing, Vol. 330, 2019, pp. 116-126.

24. L i n, H., R. K o n g, J. L i u. Genetic Algorithm Based Clustering for Large-Scale Sensor Networks. - Cybernetics and Information Technologies, Vol. 15, 2015, No 6, Special Issue on Logistics, Informatics and Service Science, pp. 168-177.

25. M i t c h e 11, M. An Introduction to Genetic Algorithms. Cambridge, MA, USA, MIT Press, 1998.

26. P a t $\mathrm{n}$ a i k, P. R. Neural Network Designs for Poly-b-Hydroxybutyrate Production Optimization under Simulated Industrial Conditions. - Biotechnology Letters, Vol. 27, 2005, pp. 409-415.

27. P e i, X., Y. Zh o u, N. W a n g. A Gaussian Process Regression Based on Variable Parameters Fuzzy Dominance Genetic Algorithm for B-TFPMM Torque Estimation. - Neurocomputing, Vol. 335, 2019, pp. 153-169.

28. Ponticelli, G. S., S. Guarino, V. Tagli a ferri, O. Giannini. An Optimized FuzzyGenetic Algorithm for Metal Foam Manufacturing Process Control. - International Journal of Advanced Manufacturing Technology, Vol. 101, 2019, No 1-4, pp. 603-614.

29. P o p o v a, S. On-line State and Parameters Estimation Based on Measurements of the Glucose in Mixed Culture System. - Biotechnology and Biotechnological Equipment, Vol. 20, 2006, No 3, pp. 208-214.

30. P o p o v a, S. Adaptive Control for PHB Production. - Acta Universitasis Cibernesis, Series E, Food Technology, Vol. XI, 2007, pp. 17-25. 
31. R o e va, O. Genetic Algorithms for a Parameter Estimation of a Fermentation Process Model: A Comparison. - Bioautomation, Vol. 3, 2005, pp. 19-28.

32. S a r a s v at h i, V., N. C. S. N. I y e n g a r, S. S a h a. QoS Guaranteed Intelligent Routing Using Hybrid PSO-GA in Wireless Mesh Networks. - Cybernetics and Information Technologies, Vol. 15, 2015, No 1, pp. 69-83.

33. S e i s in g, R., E. Trill a s, J. K a c przy k. Towards the Future of Fuzzy Logic. Switzerland, Springer, International Publishing, 2015.

34. S i d d i q u e, N. Intelligent Control. Springer, Switzerland, International Publishing, 2014.

35. S o n i k a, J. A. Genetic Algorithm Approach for Optimization of Biomass Estimation at LiDAR. In: D. Mishra, X. S. Yang, A. Unal, Eds. Data Science and Big Data Analytics, Lecture Notes on Data Engineering and Communications Technologies, Vol. 16, 2019, pp. 11-27.

36. To h y m a, M., T. P a t a rin ska, Z. Q i an g, K. S h i mizu. Modeling of the Mixed Culture and Periodic Control for PHB Production. - Biochemical Engineering Journal, Vol. 10, 2002, pp. 157-173.

37. Y a g e r, R. R., L. A. Z a d e h. An Introduction to Fuzzy Logic Applications in Intelligent Systems. Kluwer Academic Publishers, 1992.

38. Y a n g, H., D. L u o. Acyclic Real-Time Traffic Signal Control Based on a Genetic Algorithm. Cybernetics and Information Technologies, Vol. 13, 2013, No 3, pp. 111-123.

39. Z a d e h, L. Fuzzy Sets. - Information and Control, Vol. 8, 1965, pp. 338-353.

Received: 09.11.2018; Second Version: 01.04.2019; Accepted: 15.04.2019 